\title{
BIFURCATION OF POSITIVE AND NEGATIVE SOLUTIONS OF NONLINEARIZABLE STURM-LIOUVILLE PROBLEMS WITH INDEFINITE WEIGHT
}

\author{
ZIYATKHAN S. ALIYEV AND LEYLA V. NASIROVA (ASHUROVA) \\ Received 02 March, 2019
}

\begin{abstract}
We consider nonlinearizable Sturm-Liouville problem indefinite weight function. We show the existence of two pairs of global continua emanating from the bifurcation intervals surrounding the principal eigenvalues of the corresponding linear problem and contained in the classes of positive and negative functions.
\end{abstract}

2010 Mathematics Subject Classification: 34B15; 34B24; 34C23; 47J10; 47J15

Keywords: nonlinearizable Sturm-Liouville problems, indefinite weight function, bifurcation point, global continua

\section{INTRODUCTION}

We consider the following nonlinear Sturm-Liouville problem

$$
\begin{aligned}
(\ell y) \equiv-\left(p(x) y^{\prime}\right)^{\prime}+q(x) y=\lambda \rho(x) y+f\left(x, y, y^{\prime}, \lambda\right), x \in(0,1), \\
\alpha_{0} y(0)-\beta_{0} y^{\prime}(0)=0, \\
\alpha_{1} y(1)+\beta_{1} y^{\prime}(1)=0,
\end{aligned}
$$

where $\lambda \in \mathbb{R}$ is a spectral parameter, $p(x)$ is a positive continuously differentiable function on $[0,1], q(x)$ is nonnegative continuous function on $[0,1]$, and $\rho(x)$ are real-valued continuous functions on $[0,1]$ such that meas $\{x \in[0,1]: \sigma \rho(x)>0\}>0$ for each $\sigma \in\{+,-\}, \alpha_{i}, \beta_{i}, i=0,1$, are real constants such that $\left|\alpha_{i}\right|+\left|\beta_{i}\right|>0$ and $\alpha_{i} \beta_{i} \geq 0, i=0,1$. We also assume that the nonlinear term $f \in C\left([0,1] \times \mathbb{R}^{3}\right)$ is a real-valued function satisfying the following conditions:

$$
u f(x, u, s, \lambda) \leq 0
$$

there exists constants $K>0$ such that

$$
\left|\frac{f(x, u, s, \lambda)}{u}\right| \leq K,(x, u, s, \lambda) \in[0,1] \times \mathbb{R}^{3} .
$$


Problem (1.1)-(1.3) with $\rho>0$ in a more general case has been considered in $[6,7,10,11]$, where it is proved that there exist global continua of nontrivial solutions in $\mathbb{R} \times C^{1}[0,1]$ (which is a Banach space with the norm defined by $\|(\lambda, u)\|=$ $\left\{|\lambda|^{2}+\|u\|_{1}^{2}\right\}^{\frac{1}{2}}$, where $\|u\|_{1}=\|u\|_{\infty}+\left\|u^{\prime}\right\|_{\infty},\|u\|_{\infty}=\max _{x \in[0,1]}|u(x)|$, is the usual norm in a Banach space $C^{1}[0,1]$ of real-valued, continuously differentiable functions on $[0,1])$ corresponding to the usual nodal properties and emanating from bifurcation points and intervals surrounding the eigenvalues of the linear problem (1.1)-(1.3) with $f \equiv 0$. Similar problems for the nonlinear eigenvalue problems of ordinary differential equations of fourth order have been considered in [1,2].

In the case when nonlinear term $f$ satisfies $o\left(|u|+\left|u^{\prime}\right|\right)$ condition near $\left(u, u^{\prime}\right)=$ $(0,0)$ problem (1.1)-(1.3) was investigated in a recent paper [5], where it was shown that there exist four families of global continua of nontrivial solutions in $\mathbb{R} \times C^{1}[0,1]$ corresponding to the usual nodal properties and emanating from bifurcation points corresponding to the eigenvalues of the linear problem (1.1)-(1.3) with $f \equiv 0$. Similar results in the nonlinear eigenvalue problems for ordinary differential equations of fourth order and elliptic partial differential equations with indefinite weights in the classes of positive and negative functions obtained in recent papers [3, 4$]$.

In the present paper we show the existence of four global continua of solutions of problem (1.1)-(1.3) emanating from principal eigenvalues of linear problem obtained from (1.1)-(1.3) by setting $f \equiv 0$ and containing in the classes of positive and negative functions.

\section{PRELiminary}

In the case of $f \equiv 0$ from (1.1)-(1.3) we obtain the corresponding linear problem

$$
\left\{\begin{array}{l}
\ell(y)(x)=\lambda \rho(x) y(x), x \in(0,1), \\
y \in \text { B.C. }
\end{array}\right.
$$

where by B.C. we denote the set of boundary conditions (1.2), (1.3). It is a classical result (see $[8,9]$ ) that the problem $(2.1)$ in the case $\rho(x)>0, x \in[0,1]$, possesses infinitely many real eigenvalues

$$
\lambda_{1}<\lambda_{2}<\cdots<\lambda_{k}<\cdots,
$$

all of which are simple and $\lim _{k \rightarrow+\infty} \lambda_{k}=+\infty$. The eigenfunction $y_{k}(x)$ corresponding to eigenvalue $\lambda_{k}, k \in \mathbb{N}$, has exactly $k-1$ simple zeros in the interval $(0,1)$.

Let $E=C^{1}[0,1] \cap B . C$. be a Banach space with the norm $\|u\|_{1}$. Let $S_{k}^{+}$be the set of $u \in E$ which have exactly $k-1$ simple zeros in $(0,1)$ and which are positive for $0 \neq x$ near 0 ; then, $S_{k}^{-}=-S_{k}^{+}$and $S_{k}=S_{k}^{-} \cup S_{k}^{+}$. The sets $S_{k}^{+}, S_{k}^{-}$and $S_{k}$ are open in $E$. Moreover, if $u \in \partial S_{k}$, then $u$ has at least one double zero in $[0,1]$. 
Theorem 1 (see [9], Ch. 10, $\S \S 10 \cdot 6,10 \cdot 61$ ). The eigenvalues of problem (2.1) are all real and simple, and form a two sequences

$$
0>\lambda_{1}^{-}>\lambda_{2}^{-}>\ldots>\lambda_{k}^{-} \mapsto-\infty \text { and } 0<\lambda_{1}^{+}<\lambda_{2}^{+}<\ldots<\lambda_{k}^{+} \mapsto+\infty .
$$

Moreover, for each $k \in \mathbb{N}$ and each $\sigma \in\{+,-\}$ the eigenfunction $y_{k}^{\sigma}(x)$ corresponding to eigenvalue $\lambda_{k}^{\sigma}$, has exactly $k-1$ simple zeros in the interval $(0,1)$ (more precisely, $\left.y_{k}^{\sigma}(x) \in S_{k}\right)$.

We define the linear differential operator $L: D(L) \rightarrow L_{2}(0,1)$ by

$$
(L y)(x)=(\ell y)(x),
$$

and

$$
D(L)=\left\{y \in L_{2}(0,1): y \in W_{2}^{2}(0,1), \ell y \in L_{2}(0,1), y \in B . C .\right\} .
$$

It is easy to see that the differential operator $L$ is a densely defined self-adjoint operator on $L_{2}(0,1)$ whose spectrum contains only positive eigenvalues. For fixed $\lambda \in \mathbb{R}$ we consider the following eigenvalue problem

$$
\left\{\begin{array}{l}
(\ell y)(x)-\lambda \rho(x) y(x)=\mu y(x), x \in(0,1), \\
y \in \text { B.C. }
\end{array}\right.
$$

It is well known that for each $\lambda \in \mathbb{R}$ the eigenvalues problem (2.2) are real, simple and forms a infinitely increasing sequence

$$
\mu_{1}(\lambda)<\mu_{2}(\lambda)<\cdots<\mu_{k}(\lambda)<\cdots .
$$

Moreover, for each $k \in \mathbb{N}$ the eigenfunction $y_{k}(x, \lambda)$ corresponding to the eigenvalue $\mu_{k}(\lambda)$ has $k-1$ simple zeros in $(0,1)$ (it should be noted that $\left.y_{k}(x, \lambda) \in S_{k}\right)$.

For each $k \in \mathbb{N}$ the $k$-th eigenvalue $\mu_{k}(\lambda), k \in \mathbb{N}$, of problem (2.2) can be characterized as

$$
\mu_{k}(\lambda)=\max _{V^{(k-1)}} \min _{y \in B . C .}\left\{R_{\lambda}[y]: \int_{0}^{1} y(x) \varphi(x) d x=0, \varphi \in V^{(k-1)}\right\},
$$

where

$$
\begin{gathered}
R_{\lambda}[y]=\frac{\int_{0}^{1}\left\{y^{\prime 2}+q(x) y^{2}\right\} d x+N[y]}{\int_{0}^{1} y^{2} d x}-\frac{\lambda \int_{0}^{1} \rho(x) y^{2} d x}{\int_{0}^{1} y^{2} d x}, \\
N[y]=-y^{\prime}(1) y(1)+y^{\prime}(0) y(0) \geq 0,
\end{gathered}
$$

and $V^{(k-1)}$ denotes any set of $(k-1)$ linearly independent functions with $\varphi_{j}(x) \in$ B.C., $1 \leq j \leq k-1$ (see [8]). From this max-min characterization it follows that the number $\lambda$ is an eigenvalue of problem (2.1) corresponding to the eigenfunction having $k-1$ simple zeros in $(0,1)$, if and only if $\mu_{k}(\lambda)=0$. Moreover for each $k \in \mathbb{N}$ 
the eigenvalue $\mu_{k}(\lambda)$ of problem (2.2) and corresponding eigenfunction $y_{k}(x, \lambda)$ are continuous functions of $\lambda \in \mathbb{R}$.

In order to study the local and global bifurcation of solutions of problems (1.1)(1.3) in the classes of positive and negative functions we need the following properties of eigenvalues $\mu_{1}(\lambda)$ as a functions of $\lambda \in \mathbb{R}$.

Lemma 1. Let $y_{k}(x, \lambda), k \in \mathbb{N}$, be an eigenfunction of (2.2) corresponding to the $k$-th eigenvalue $\mu_{k}(\lambda)$. Then $\mu_{k}(\lambda) \in C^{\infty}(\mathbb{R})$ and

$$
\frac{d \mu_{k}(\lambda)}{d \lambda}=-\frac{\int_{0}^{1} \rho(x) y_{k}^{2}(x, \lambda) d x}{\int_{0}^{1} y_{k}^{2}(x, \lambda) d x}, \lambda \in \mathbb{R}, k \in \mathbb{N} .
$$

Proof. By virtue of (2.2), for each $k \in \mathbb{N}$ and $v, \lambda \in \mathbb{R}$, we have

$$
\begin{gathered}
y_{k}(x, \lambda) \ell y_{k}(x, v)-y_{k}(x, v) \ell y_{k}(x, \lambda)-(v-\lambda) \rho(x) y_{k}(x, v) y_{k}(x, \lambda)= \\
\left(\mu_{k}(v)-\mu_{k}(\lambda)\right) y_{k}(x, v) y_{k}(x, \lambda), x \in(0,1) .
\end{gathered}
$$

Integrating this relation from 0 to 1 , using the formula for the integration by parts, and taking into account conditions (1.2), (1.3), we obtain

$$
\begin{aligned}
& \left(\mu_{k}(\mathrm{v})-\mu_{k}(\lambda)\right) \int_{0}^{1} y_{k}(x, v) y_{k}(x, \lambda) d x= \\
& -(v-\lambda) \int_{0}^{1} \rho(x) y_{k}(x, v) y_{k}(x, \lambda) d x .
\end{aligned}
$$

For $v, \lambda \in \mathbb{R}, v \neq \lambda$, we get

$$
\frac{\mu_{k}(v)-\mu_{k}(\lambda)}{v-\lambda}=-\frac{\int_{0}^{1} \rho(x) y_{k}(x, v) y_{k}(x, \lambda) d x}{\int_{0}^{1} y_{k}(x, v) y_{k}(x, \lambda) d x} .
$$

Note that $y_{k}(x, v) \rightarrow y_{k}(x, \lambda)$ as $v \rightarrow \lambda$ uniformly in $x \in[0,1]$ (see [9], Ch. 10, $\S 10 \cdot 72$ ). Then passing to the limit as $v \rightarrow \lambda$ in (2.8) we obtain (2.5). Moreover, $\mu_{k}(\lambda) \in C^{1}(\mathbb{R})$. Next, by the principle of mathematical induction, it is proved that $\mu_{k}(\lambda) \in C^{\infty}(\mathbb{R})$.

Lemma 2. The function $\lambda \rightarrow \mu_{1}(\lambda)$ is a concave in $\mathbb{R}$. 
Proof. By virtue of Lemma 1 differentiating (2.5) we get

$$
\begin{gathered}
\frac{d^{2} \mu_{1}(\lambda)}{d \lambda^{2}}=-2\left(\int_{0}^{1} y_{1}^{2}(x, \lambda) d x\right)^{-2} \\
\quad \int_{0}^{1} \rho(x) y_{1}(x, \lambda) \frac{\partial y_{1}(x, \lambda)}{\partial \lambda} d x \int_{0}^{1} y_{1}^{2}(x, \lambda) d x- \\
\left.-\int_{0}^{1} \rho(x) y_{1}^{2}(x, \lambda) d x \int_{0}^{1} y_{1}(x, \lambda) \frac{\partial y_{1}(x, \lambda)}{\partial \lambda} d x\right\}= \\
\left.\int_{0}^{1} \rho(x) y_{1}(x, \lambda) \frac{\partial y_{1}(x, \lambda}{\partial \lambda}\right) d x+\frac{d \mu_{1}(\lambda)}{d \lambda} \int_{0}^{1} y_{1}(x, \lambda) \frac{d y_{1}(x, \lambda)}{d \lambda} d x \\
\int_{0}^{1} y_{1}^{2}(x, \lambda) d x
\end{gathered} .
$$

On the other hand, by Lemma 1 from (2.2) we have the following relation

$$
\begin{gathered}
\ell\left(\frac{\partial y_{1}(x, \lambda)}{\partial \lambda}\right)-\lambda \rho(x) \frac{\partial y_{1}(x, \lambda)}{\partial \lambda}-\rho(x) y_{1}(x, \lambda)= \\
\mu_{1}(\lambda) \frac{\partial y_{1}(x, \lambda)}{\partial \lambda}+\frac{d \mu_{1}(\lambda)}{d \lambda} y_{1}(x, \lambda) .
\end{gathered}
$$

Multiplying both sides of equation (2.10) by $\frac{\partial y_{1}(x, \lambda)}{\partial \lambda}$ and integrating the obtained equality from 0 to 1 , using the formula for the integration by parts, and taking into account boundary conditions (1.2), (1.3), we obtain

$$
\begin{gathered}
\int_{0}^{1}\left(\frac{\partial y_{1}^{\prime}(x, \lambda)}{\partial \lambda}\right)^{2} d x+\int_{0}^{1} q(x)\left(\frac{\partial y_{1}(x, \lambda)}{\partial \lambda}\right)^{2} d x+ \\
N\left[\frac{\partial y_{1}(x, \lambda)}{\partial \lambda}\right]-\lambda \int_{0}^{1} \rho(x)\left(\frac{\partial y_{1}(x, \lambda)}{\partial \lambda}\right)^{2} d x- \\
\int_{0}^{1} \rho(x) y_{1}(x, \lambda) \frac{\partial y_{1}(x, \lambda)}{\partial \lambda} d x=\mu_{1}(\lambda) \int_{0}^{1}\left(\frac{\partial y_{1}(x, \lambda)}{\partial \lambda}\right)^{2} d x+ \\
\frac{d \mu_{1}(\lambda)}{d \lambda} \int_{0}^{1} y_{1}(x, \lambda) \frac{\partial y_{1}(x, \lambda)}{\partial \lambda} d x .
\end{gathered}
$$


By virtue of (2.9) it follows from (2.11) that

$$
\begin{gathered}
\int_{0}^{1}\left(\frac{\partial y_{1}^{\prime}(x, \lambda)}{\partial \lambda}\right)^{2} d x+\int_{0}^{1} q(x)\left(\frac{\partial y_{1}(x, \lambda)}{\partial \lambda}\right)^{2} d x+ \\
N\left[\frac{\partial y_{1}(x, \lambda)}{\partial \lambda}\right]-\lambda \int_{0}^{1} \rho(x)\left(\frac{\partial y_{1}(x, \lambda)}{\partial \lambda}\right)^{2} d x- \\
\mu_{1}(\lambda) \int_{0}^{1}\left(\frac{\partial y_{1}(x, \lambda)}{\partial \lambda}\right)^{2} d x=-\frac{1}{2} \frac{d^{2} \mu_{1}(\lambda)}{d \lambda^{2}} \int_{0}^{1} y_{1}^{2}(x, \lambda) d x
\end{gathered}
$$

which implies that

$$
\begin{aligned}
& \frac{d^{2} \mu_{1}(\lambda)}{d \lambda^{2}}= \\
& -2\left\{\frac{\int_{0}^{1}\left(\frac{\partial y_{1}^{\prime}(x, \lambda)}{\partial \lambda}\right)^{2} d x+\int_{0}^{1} q(x)\left(\frac{\partial y_{1}(x, \lambda)}{\partial \lambda}\right)^{2} d x+N\left[\frac{\partial y_{1}(x, \lambda)}{\partial \lambda}\right]}{\int_{0}^{1}\left(\frac{\partial y_{1}(x, \lambda)}{\partial \lambda}\right)^{2} d x}-\right. \\
& \left.\frac{\lambda \int_{0}^{1} \rho(x)\left(\frac{\partial y_{1}(x, \lambda)}{\partial \lambda}\right)^{2} d x}{\int_{0}^{1}\left(\frac{\partial y_{1}(x, \lambda)}{\partial \lambda}\right)^{2} d x}-\mu_{1}(\lambda)\right\} \frac{\int_{0}^{1}\left(\frac{\partial y_{1}(x, \lambda)}{\partial \lambda}\right)^{2} d x}{\int_{0}^{1} y_{1}^{2}(x, \lambda) d x} .
\end{aligned}
$$

Hence by (2.3) and (2.4) from (2.12) we get $\frac{d^{2} \mu_{1}(\lambda)}{d \lambda^{2}}<0$ for $\lambda \in \mathbb{R}$.

\section{Global bifurcation of Positive AND NEgative Solutions of} PROBLEM (1.1)-(1.3)

Along with (2.1) and (2.2) we consider the following linear eigenvalue problems

$$
\begin{gathered}
\left\{\begin{array}{l}
(\ell y)(x)+\psi(x) y(x)=\lambda \rho(x) y(x), x \in(0,1), \\
y \in B . C .,
\end{array}\right. \\
\left\{\begin{array}{l}
(\ell y)(x)+\psi(x) y(x)-\lambda \rho(x) y(x)=\mu y(x), x \in(0,1), \\
y \in \text { B.C., }
\end{array}\right.
\end{gathered}
$$

where $\psi$ is a nonnegative continuous function on $[0,1]$.

Since $\psi(x) \geq 0, x \in[0,1]$, it follows from formula (4.2) of [2] that

$$
0 \leq \tilde{\mu}_{k}(\lambda)-\mu_{k}(\lambda) \leq \tilde{K}
$$


where $\tilde{\mu}_{k}(\lambda)$ is the $k$-th eigenvalue of problem (3.2) and $\tilde{K}=\sup \{\psi(x): x \in[0,1]\}$.

Remark 1 . Since the function $\hat{\mu}_{1}(\lambda)$ is also a concave on $\mathbb{R}$ and $\tilde{\mu}_{1}(\lambda) \geq \mu_{k}(\lambda)$ for any $\lambda \in \mathbb{R}$ it follows that $\tilde{\lambda}_{1}^{+}>\lambda_{1}^{+}$and $\tilde{\lambda}_{1}^{-}<\lambda_{1}^{-}$, where $\tilde{\lambda}_{1}^{+}$and $\tilde{\lambda}_{1}^{-}$are the positive and negative principal eigenvalues of problem (3.2), respectively.

In what follows we need the following result.

Lemma 3. For each $\sigma \in\{+,-\}$ the following relation holds:

$$
\left|\tilde{\lambda}_{1}^{\sigma}-\lambda_{1}^{\sigma}\right| \leq \frac{\sigma \int_{0}^{1}\left(y_{1}^{\sigma}(x, \lambda)\right)^{2} d x}{\int_{0}^{1} \rho(x)\left(y_{1}^{\sigma}(x, \lambda)\right)^{2} d x} .
$$

The proof of this lemma is similar to that of Lemma 3.5 of [4].

Remark 2. Since the class of continuous functions $C[0,1]$ is dense in $L_{1}[0,1]$ the statement of Lemma 3 holds for $\psi \in L_{1}[0,1]$.

In order to study the bifurcation of solutions of problem (1.1)-(1.3) we define the sets $S_{k}^{\sigma, v}, k \in \mathbb{N}, S_{k, \sigma}, \sigma \in\{+,-\}$ and $v \in\{+,-\}$, as follows:

$$
S_{k}^{\sigma, v}=\left\{y \in S_{k}^{v}: \sigma \int_{0}^{1} \rho(x) y^{2}(x) d x>0\right\}, S_{k, \sigma}=S_{k}^{\sigma,+} \cup S_{k}^{\sigma,-} .
$$

Remark 3. It follows immediately from the definition of the sets $S_{k}^{\sigma, v}$ and $S_{k, \sigma}$, $k \in \mathbb{N}, \sigma, v \in\{+,-\}$, that these sets are open subsets of $E$ and $S_{k}^{\sigma, v} \cap S_{m}^{\rho, \theta}=\varnothing$, $S_{k, \sigma} \cap S_{m, \rho}=\varnothing$, where $(k, \sigma, v) \neq(m, \rho, \theta),(k, \sigma) \neq(m, \rho)$, respectively. Moreover, if $y$ lies of the boundary of some $S_{k, \sigma}$, then either $y$ must have a double zero in $[0,1]$ or $\int_{0}^{1} \rho(x) y^{2}(x) d x=0$.

The norm in $\mathbb{R} \times E$ is defined to be $\|(\lambda, u)\|=\left\{|\lambda|^{2}+\|u\|_{1}^{2}\right\}^{\frac{1}{2}}$.

We denote by $C$ the closure in $\mathbb{R} \times E$ of the set of nontrivial solutions of (1.1)-(1.3). The eigenfunction $y_{k}^{\sigma}, \sigma \in\{+,-\}$, corresponding to the eigenvalue $\lambda_{k}^{\sigma}$ of problem (2.1) is made unique by requiring that $y_{k}^{\sigma} \in S_{k}^{\sigma,+}$ and $\left\|y_{k}^{\sigma,+}\right\|_{1}=1$.

Lemma 4. If $(\lambda, y) \in \mathbb{R} \times E$ is a solution of (1.1)-(1.3) such that $y \in \partial S_{k}^{\sigma, v}, k \in$ $\mathbb{N}, \sigma, v \in\{+,-\}$, then $y \equiv 0$.

Proof. Let $(\lambda, y)$ be a solution of (1.1)-(1.3) and let $y \in \partial S_{k}^{\sigma, v}$. Then by Remark 3 either there exists a point $\xi \in[0,1]$ such that $y(\xi)=y^{\prime}(\xi)=0$ or $\int_{0}^{1} \rho(x) y^{2}(x) d x=0$. 
Suppose that $y \in \partial S_{k}^{\sigma, v}$ and $y(\xi)=y^{\prime}(\xi)=0$ for some $\xi \in[0,1]$. Then, using growth estimate on $f$ and linearity of $\ell$ and $\rho y$, and applying Gronwall's inequality we obtain that $y \equiv 0$ on $[0,1]$ (see Lemma 3.1 of [2]).

Now let $y \in \partial S_{k}^{\sigma, v}$ and $\int_{0}^{1} \rho(x) y^{2}(x) d x=0$. Then by (1.1)-(1.3) we have

$$
\int_{0}^{1}\left(y^{\prime 2}+q(x) y^{2}\right) d x+N[y]=\int_{0}^{1} y(x) h\left(x, y(x), y^{\prime}(x), \lambda\right) d x .
$$

Since the left-hand side of this equality is nonnegative (see inequality after formula (2.4)) and the right-hand side is nonpositive in view of (1.4), it follows that $y \equiv 0$.

Corollary 1. Suppose that the condition (1.4) is not satisfied. If $(\lambda, y) \in \mathbb{R} \times E$ is a solution of 1.1)-(1.3) such that $y \in \partial S_{k}^{v}, k \in \mathbb{N}, v \in\{+,-\}$, then $y \equiv 0$.

We say that $(\lambda, 0), \lambda \in \mathbb{R}$, is a bifurcation point of (1.1)-(1.3) with respect to the set $\mathbb{R} \times S_{k}^{\sigma, v}\left(\mathbb{R} \times S_{k, \sigma}\right)$ if in every small neighborhood of this point there is a solution of this problem which is contained in $\mathbb{R} \times S_{k}^{\sigma, v}\left(\mathbb{R} \times S_{k}^{\sigma}\right)$ (see [2]).

Along with (1.1)-(1.3) we consider the following approximating problem

$$
\left\{\begin{array}{l}
\ell y(x)=\lambda \rho(x) y(x)+f\left(x,|y|^{\varepsilon} y, y^{\prime}, \lambda\right), x \in(0,1), \\
y \in B . C .
\end{array}\right.
$$

where $\varepsilon \in(0,1]$.

By virtue of boundary condition (1.3) we have

$$
f\left(x,|u|^{\varepsilon} u, v, \lambda\right)=o(|u|+|s|) \text { as }|u|+|s| \rightarrow 0,
$$

uniformly in $x \in[0,1]$ and $\lambda \in \mathbb{R}$. Then it follows from Theorem 2 of [5] that for each $k \in \mathbb{N}$, each $\sigma \in\{+,-\}$ and each $v \in\{+,-\}$ there exists an unbounded continuum $D_{k}^{\sigma, v}$ of solutions of problem (3.5) in $\left(\mathbb{R} \times S_{k}^{\sigma, v}\right) \cup\left\{\left(\lambda_{k}^{\sigma}, 0\right)\right\}$ which contain $\left(\lambda_{k}^{\sigma}, 0\right)$ and is unbounded in $\mathbb{R} \times E$. Moreover, by condition (1.4) if $(\lambda, y) \in D_{k}^{\sigma, v}$, then $\sigma \lambda>0$.

Lemma 5. For each $\sigma \in\{+,-\}, v \in\{+,-\}$ and each $0<r<1$ problem (1.1)(1.3) has a solution $\left(\lambda_{1, r}^{\sigma, v}, y_{1, r}^{\sigma, v}\right)$ such that $\left\|y_{1, r}^{\sigma, v}\right\|=r, y_{1, r}^{\sigma, v} \in S_{1}^{\sigma, v}$ and $\lambda_{1, r}^{\sigma, v} \in I_{1}^{\sigma}$, where $I_{1}^{+}=\left[\lambda_{1}^{+}, \lambda_{1}^{+}+h_{1}^{+}\right], I_{1}^{-}=\left[\lambda_{1}^{-}-h_{1}^{-}, \lambda_{1}^{-}\right]$and

$$
h_{1}^{+}=\frac{\int_{0}^{1}\left(y_{1}^{+}(x)\right)^{2} d x}{\int_{0}^{1} \rho(x)\left(y_{1}^{+}(x)\right)^{2} d x}, h_{1}^{-}=-\frac{\int_{0}^{1}\left(y_{1}^{-}(x)\right)^{2} d x}{\int_{0}^{1} \rho(x)\left(y_{1}^{-}(x)\right)^{2} d x} .
$$


Proof. Let $\left(\lambda_{\varepsilon}, y_{\varepsilon}\right)$ be a solution of problem (3.5) such that $\lambda_{\varepsilon}>0, y_{\varepsilon} \in S_{1}^{v}$ and $\left\|y_{\varepsilon}\right\|<1$. We define a function $\psi_{\varepsilon}(x), x \in[0,1]$, as follows:

$$
\psi_{\varepsilon}(x)=\left\{\begin{array}{cl}
-\frac{f\left(x,\left|y_{\varepsilon}(x)\right|{ }^{\mid} y_{\varepsilon}(x), y_{\varepsilon}^{\prime}(x), \lambda_{\varepsilon}\right)}{y_{\varepsilon}(x)} & \text { if } y_{\varepsilon}(x) \neq 0, \\
0 & \text { if } y_{\varepsilon}(x)=0 .
\end{array} .\right.
$$

Then $\left(\lambda_{\varepsilon}, y_{\varepsilon}\right)$ is a solution of the following spectral problem

$$
\left\{\begin{array}{l}
\ell y(x)+\psi_{\varepsilon}(x) y(x)=\lambda \rho(x) y(x), x \in(0,1), \\
y \in \text { B.C. }
\end{array}\right.
$$

Since $y_{\varepsilon}(x) \neq 0$ for $x \in(0,1)$ it follows that $\lambda_{\varepsilon}$ is a positive principal eigenvalue of problem (3.7).

By virtue of ||$y_{\varepsilon} \|_{1}<1$ we have $\left|y_{\varepsilon}(x)\right|<1$. Then by (1.4) and (1.5) from (3.6) we obtain the following relations

$$
\psi_{\varepsilon}(x) \geq 0 \text { and }\left|\psi_{\varepsilon}(x)\right| \leq K|y(x)|^{\varepsilon} \leq K, x \in[0,1] .
$$

Note that $y_{\varepsilon}(x) \neq 0$ for $x \in(0,1)$ and is bounded on the closed interval $[0,1]$. Then it follows from Remark 2 that the statement of Lemma 3 holds for the problem (3.7). Hence by (3.8) from (3.4) we obtain

$$
\left|\lambda_{\varepsilon}-\lambda_{1}^{+}\right|<h_{1}^{+}
$$

with implies that $\lambda_{\varepsilon} \in I_{1}^{+}$. In the case when $\lambda_{\varepsilon}<0$ we have $\lambda_{\varepsilon} \in I_{1}^{-}$.

Let $\left\{\varepsilon_{n}\right\}_{n=1}^{\infty}, 0<\varepsilon_{n}<1$, be a sequence converging to zero. Since the continuum $D_{1}^{\sigma, v}$ of solutions of problem (3.5) containing the point $\left(\lambda_{1}^{\sigma}, 0\right)$ is unbounded in $\mathbb{R} \times E$, it follows that for each $\varepsilon_{n}$ and each $r \in(0,1)$ problem (3.5) has a solution $\left(\lambda_{1, r, \varepsilon_{v}}^{\sigma, v}, y_{1, r, \varepsilon_{n}}^{\sigma, v}\right)$ such that $\lambda_{1, r, \varepsilon_{n}}^{\sigma, v} \in I_{1}^{\sigma}, y_{1, r, \varepsilon_{n}}^{\sigma, v} \in S_{1}^{\sigma, v}$ and $\left\|y_{1, r, \varepsilon_{n}}^{\sigma, v}\right\|_{1}=r$. Since the sequence $\left\{y_{1, r, \varepsilon_{n}}^{\sigma, v}\right\}_{n=1}^{\infty}$ is bounded in $C^{1}[0,1], f$ is continuous in $[0,1] \times \mathbb{R}^{3}$, and the set $\left\{\lambda_{1, r, \varepsilon_{n}}^{\sigma, v}\right\}_{n=1}^{\infty}$ is bounded in $\mathbb{R}$, it follows from 3.5) that $\left\{y_{1, r, \varepsilon_{n}}^{\sigma, v}\right\}_{n=1}^{\infty}$ is also bounded in $C^{2}[0,1]$. Then by Arzelà-Ascoli theorem we can assume (without loss of generality) that $\left\{y_{1, r, \varepsilon_{n}}^{\sigma, v}\right\}_{n=1}^{\infty}$ converges in $E$ to a function $y_{1, r, v}^{\sigma, v}$ with $\left\|y_{1, r}^{\sigma, v}\right\|_{1}=r$. Without loss of generality we can also assume that $\left\{\lambda_{1, r, \varepsilon_{n}}^{\sigma, v}\right\}_{n=1}^{\infty}$ converges to some $\lambda_{1, r}^{\sigma, v} \in I_{1}^{\sigma}$. Next passing to the limit in (3.5) at $n \rightarrow \infty$, we get that $\left(\lambda_{1, r}^{\sigma, v}, y_{1, r}^{\sigma, v}\right)$ is a solution of problem (1.1)-(1.3). Since $y_{1, r}^{\sigma, v}$ is contained in the closure of the set $S_{1}^{\sigma, v}$ and $\left\|y_{1, r}^{\sigma, v}\right\|_{1}=r>0$, it follows from Lemma 4 that $y_{1, r}^{\sigma, v} \in S_{1}^{\sigma, v}$.

Corollary 2. The set of bifurcation points of problem (1.1)-(1.3) is nonempty. If $(\lambda, 0)$ is a bifurcation point of problem (1.1)-(1.3) with respect to the set $S_{1}^{\sigma, v}$, then $\lambda \in I_{1}^{\sigma}$.

For $\sigma \in\{+,-\}$ and $v \in\{+,-\}$ let $\tilde{D}_{1}^{\sigma, v}$ denote the union of connected components $D_{1, \lambda}^{\sigma, v}$ of the solution set of problem (1.1)-(1.3) emanating from bifurcation points $(\lambda, 0) \in I_{1}^{\sigma} \times 0$ with respect to the set $S_{1}^{\sigma, v}$. It follows from Corollary 2 that $\tilde{D}_{1}^{\sigma, v} \neq \varnothing$. 
Note that $D_{1}^{\sigma, v}=\tilde{D}_{1}^{\sigma, v} \cup\left(I_{1}^{\sigma} \times\{0\}\right)$ is a connected subset of $\mathbb{R} \times E$, but $\tilde{D}_{1}^{\sigma, v}$ is not necessarily connected in $\mathbb{R} \times E$.

The main result of this paper is the following theorem.

Theorem 2. For each $\sigma \in\{+,-\}$ and $v \in\{+,-\}$ the connected component $D_{1}^{\sigma, v}$ of the solution set of problem (1.1)-(1.3) lies in the strip $I_{1}^{\sigma} \times S_{1}^{\sigma, v}$ and is unbounded $\mathbb{R} \times E$.

Proof. Let $(\lambda, y) \in \mathbb{R} \times S_{1}^{\sigma, v}$ be a solution of problem (1.1)-(1.3). We define the function $\psi(x)$ as follows:

$$
\psi(x)=\left\{\begin{array}{cl}
-\frac{f\left(x, y(x), y^{\prime}(x), \lambda\right)}{y(x)} & \text { if } y(x) \neq 0, \\
0 & \text { if } y(x)=0 .
\end{array}\right.
$$

Then $(\lambda, y)$ is a solution of problem (3.1). By (1.4)-(1.5) it follows from (3.9) that

$$
\psi(x) \geq 0 \text { and }|\psi(x)| \leq K \text { for } x \in[0,1] .
$$

Since $y \in S_{1}^{\sigma, v}$ it follows from Theorem 1 that $\lambda$ is a principal eigenvalue of problem (3.1). Then by virtue of Lemma 3 we have $\lambda \in I_{1}^{\sigma}$. Thus we show that if $(\lambda, y) \in$ $\mathbb{R} \times S_{1}^{\sigma, v}$ is a solution of problem (1.1)-(1.3), then $\lambda \in I_{1}^{\sigma}$. Next proof of this theorem is similar to that of Theorem 1.3 of [2].

\section{ACKNOWLEDGEMENTS}

The authors are grateful to the referee for his valuable remarks and suggestions, which contributed to a significant improvement in the text and understanding of the results.

\section{REFERENCES}

[1] Z. S. Aliyev, "Some global results for nonlinear fourth order eigenvalue problems." Cent. Eur. J. Math., vol. 12, no. 12, pp. 1811-1828, 2014, doi: 10.2478/s11533-014-0416-Z.

[2] Z. S. Aliyev, "On the global bifurcation of solutions of some nonlinear eigenvalue problems for ordinary differential equations of fourth order." Sb. Math., vol. 207, no. 12, pp. 1625-1649, 2016, doi: $10.1070 /$ SM8369.

[3] Z. S. Aliyev and S. M. Hasanova, "Global bifurcation of positive solutions of semi-Linear elliptic partial differential equations with indefinite weight." Z. Anal. Anwend., vol. 38, no. 1, pp. 1-15, 2019, doi: 10.4171/ZAA/1625.

[4] Z. S. Aliyev and R. A. Huseynova, "Bifurcation in nonlinearizable eigenvalue problems for ordinary differential equations of fourth order with indefinite weight." Electron. J. Qual. Theory Differ. Equat., no. 92, pp. 1-12, 2017, doi: 10.14232/ejqtde.2017.1.92.

[5] L. V. Ashurova, "Global bifurcation of solutions for the problem of population modeling." Casp. J. Appl. Math., Ecol., Econom., vol. 5, no. 1, pp. 65-71, 2017.

[6] H. Berestycki, "On some nonlinear Sturm-Liouville problems." J. Diffential Equations, vol. 26, pp. 375-390, 1977, doi: 10.1016/0022-0396(77)90086-9.

[7] R. Chiappinelli, "On eigenvalues and bifurcation for nonlinear Sturm-Liouville operators." Boll. Un. Mat. Ital., vol. A4, no. 92, pp. 77-83, 1985, doi: 10.14232/ejqtde.2017.1.92. 
[8] R. Courant and D. Hilbert, Methoden der Mathematischen Physik, I. Berlin Heidelberg: SpringerVerlag, 1924. doi: 10.1007/978-3-642-96050-5.

[9] E. L. Ince, Ordinary Differential Equations. Berlin Heidelberg: Springer-Verlag, 1924.

[10] P. H. Rabinowitz, "Some global results for nonlinear eigenvalue problems." J. Funct. Anal., vol. 7, no. 3, pp. 487-513, 1971, doi: 10.1016/0022-1236(71)90030-9.

[11] B. P. Rynne, "Bifurcation from zero or infinity in Sturm-Liouville problems which are not linearizable.” J. Math. Anal. Appl., vol. 228, no. 1, pp. 141-156, 1998, doi: 10.1006/jmaa.1998.6122.

Authors' addresses

\section{Ziyatkhan S. Aliyev}

Baku State University, Department of Mathematical Analysis, 23 Z. Khalilov St., AZ1148 Baku, Azerbaijan

Current address: Institute of Mathematics and Mechanics NAS of Azerbaijan, Department of Differential Equations, 9 B. Vahabzadeh St., AZ1141 Baku, Azerbaijan

E-mail address: z_aliyevemail.ru

\section{Leyla V. Nasirova (Ashurova)}

Sumgait State University, Department of Mathematical Analysis, 43rd block, AZ5008 Sumgait, Azerbaijan

E-mail address: leyla.ashurova25@gmail.com 